\title{
METABOLIC STONE SCREENING - CAN EDUCATION IMPROVE ADHERENCE TO NATIONAL GUIDELINES?
}

\author{
Manoj Ravindraanandan ${ }^{1}$ and Panduka Jayawardena ${ }^{2}$ \\ ${ }^{1}$ University Hospitals of North Midlands NHS Trust, Stoke-on-Trent, UK \\ ${ }^{2}$ Royal Shrewsbury Hospital, Shrewsbury, Shropshire, England
}

Submitted: January 3, 2020. Accepted: February 2, 2020. Published: February 7, 2020.

\begin{abstract}
Nephrolithiasis is a common urological disease affecting approximately $13 \%$ of the global population. Identifying a preventable cause during follow-up for stone formation is a necessary factor in reducing recurrence.

The majority of kidney stones are comprised of calcium, with oxalate and phosphate compounds accounting for approximately $80 \%$ of stone formers. Serum analysis can be used to identify the levels of calcium in the blood, excluding metabolic causes for stones. Current NICE guidelines recommend performing a metabolic screen during an acute admission. Adherence to these guidelines from clinicians can be poor. We aim to see whether education can improve serum metabolic requests from clinicians in the UK.
\end{abstract}

\section{Method}

A case-control study was performed in a single rural district general hospital in the UK. Patients who presented with renal colic were analyzed retrospectively for four months, looking at serum metabolic screen requests. Two months of education was then delivered to acute departments, with a further prospective study performed following this for six months. Outcomes were then compared between both groups to see if there was an improvement in serum requests following the delivery of local education.

\section{Results}

A total of 124 patients were included in our study. 50 patients were analyzed in cohort A over a 4-month period. Complete serum analysis was performed on $22 \%(n=11)$ of patients. Incomplete serum analysis was performed on 58\% (n-29) of patients with uric acid being missed on all of them. 10 patients had no metabolic serum analysis performed at all, with 1 patient having a previous history of stones.

A total of 74 patients were analyzed in cohort B over a 6-month period. This was following the 2 months of departmental education given by urologists. Complete serum analysis was performed on $24 \%(n=18)$ of patients. Incomplete serum analysis was performed on $55 \%(\mathrm{n}=41)$ of patients with uric acid being missed from all of them. The remaining $21 \%$ of patients $(n=15)$ had no metabolic serum screen performed at all.

There was no significant difference seen between both cohorts after comparing metabolic serum requests before and after department education for 2 months ( $\mathrm{p}=0.7287$ ).

\section{Conclusion}

Improving adherence to clinical guidelines for metabolic screening has proved difficult with departmental teaching alone, with very little improvement achieved. Nevertheless, formal face-to-face education is advocated to reinforce knowledge in junior doctors managing renal stones. 
Nephrolithiasis is a common urological disease. It represents a significant public health problem with a lifetime prevalence of $10 \%$ in men and $7 \%$ in women. ${ }^{1}$ Although many kidney stones remain asymptomatic, patients may present with pain, infection or obstruction. ${ }^{2,3}$ Furthermore following an initial stone event, more than half of patients experience recurrent stone by 5 years. ${ }^{4}$ This can result in a high economic burden on healthcare trusts across the nation. ${ }^{5}$ Current guidelines from NICE recommend metabolic screening in those who present with renal colic, to exclude any underlying metabolic causes. ${ }^{6}$ However, clinicians frequently forget to perform such screening tests on these patients, resulting in increased investment in time and money during follow-up. We aim to see whether education to acute surgical departments helps clinicians adhere to these national guidelines and whether this can be improved.

\section{METHOD}

We performed a case-control study using a cohort of patients from a single district general hospital in the UK between August 2017 and July 2018. Patients who had presented with renal colic during this time were included in our study. Data was captured using our online clinical portal system and patient notes, analyzing those who had presented acutely either to Accident and Emergency or the Surgical Assessment Unit (SAU) with a clinical suspicion of kidney stones.

Cohort A was audited retrospectively over a 4-month period. Metabolic serum tests analyzed included calcium and phosphate (bone profile) as well as uric acid in those who had confirmation of a stone on imaging. Two months of education were then delivered to our local departments focussing on serum metabolic testing. This included face-to-face teaching, posters, and emails distributed within the trust. Cohort B was then prospectively analyzed over a 6-month period following the education received, using the same variables aforementioned. "Complete" serum analysis was considered as calcium and uric acid request, comparing to national guidelines at the time of the audit. Further variables explored included a previous history of stones, whether a stone analysis was performed, and the composition of stones.

A Fisher's exact test was used to compare the significance of results from both cohorts using the online calculator, GraphPad. ${ }^{7} \mathrm{P}<0.05$ was considered to be significant.

\section{RESULTS}

A total of 124 patients with a median age of 37 (range 27-52) were included in our study. 50 patients were analyzed in cohort A over a 4-month period. From that cohort, $24 \%(n=12)$ of patients had a previous history of stones. Complete serum analysis was performed on $22 \%(n=11)$ of patients. An incomplete serum analysis (Figure 1) was performed on 58\% $(n=29)$ of patients with uric acid being missed on all of them. A total of 10 patients had no metabolic serum analysis performed at all, with 1 patient having a previous history of stones. From cohort A, 24\% $(\mathrm{n}=12)$ had provided a stone for analysis (Figure 2). Calcium oxalate was present in all stones: $33 \%(n=4)$ of stones contained pure calcium oxalate, $58 \%(n=7)$

FIG. 1 Serum analysis.

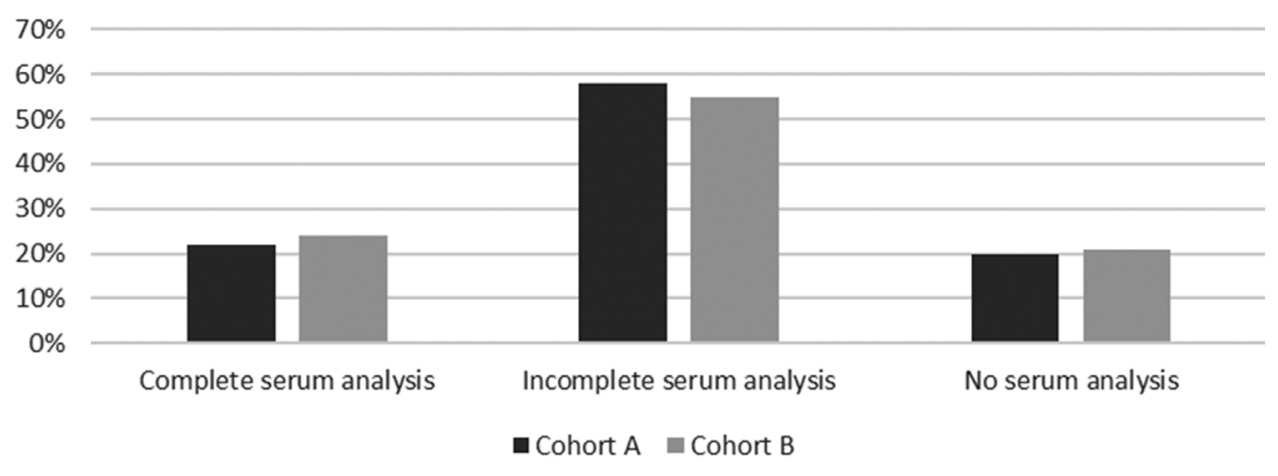

J Endolum Endourol Vol 3(1):e19-e22; February 7, 2020

This article is distributed under the terms of the Creative Commons Attribution-Non

Commercial 4.0 International License. (Ravindraanandan and Jayawardena. 
FIG. 2 Stone analysis.

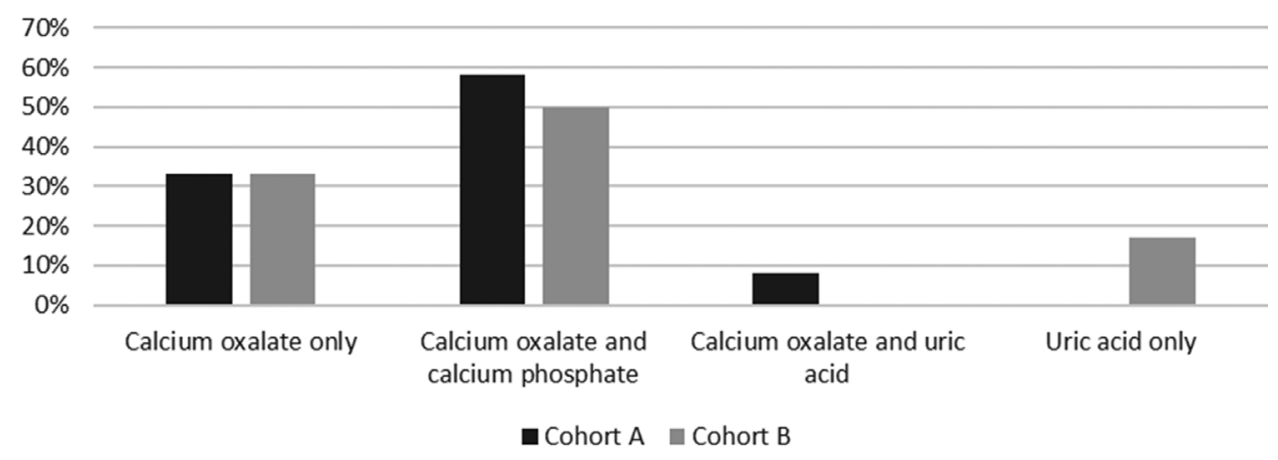

contained calcium oxalate and calcium phosphate with a predominance of the former (mean composition of oxalate $71.4 \%$ and $28.6 \%$ phosphate), and $8 \%$ of stones $(\mathrm{n}=1)$ contained calcium oxalate and uric acid ( $21 \%$ oxalate, $79 \%$ uric acid).

A total of 74 patients were analyzed in cohort B over a 6-month period. This was following the 2 months of departmental education given by urologists. From this cohort, $32 \%(n=24)$ had a previous history of stones. Complete serum analysis was performed on $24 \%(n=18)$ of patients. Incomplete serum analysis was performed on $55 \%(\mathrm{n}=41)$ of patients with uric acid being missed from all of them. The remaining $21 \%$ of patients $(n=15)$ had no metabolic serum screen performed at all, with 2 of those patients having a previous history of stones. From this cohort, only $8 \%$ of patients $(\mathrm{n}=6)$ had provided a stone for analysis. From these, $33 \%$ of stones $(n=2)$ were made from calcium oxalate alone. A mixture of calcium oxalate and phosphate made up 50\% $(n=3)$ of stones with a mean composition of oxalate $68.4 \%$ and $31.6 \%$ phosphate). There was finally 1 stone (17\%) that was made from uric acid alone.

There was no significant difference seen between both cohorts after comparing metabolic serum requests before and after department education for 2 months $(\mathrm{p}=0.7287)$.

\section{DISCUSSION}

We have demonstrated that despite formal and informal education to our local departments, the adherence rates of requesting metabolic screening tests remain the same. We believe that this could be due to a multitude of factors. Firstly, the training process for junior doctors within the United Kingdom relies on a rotational format, such as spending a limited amount of time within a department, usually four to six months, before moving on to a different department to broaden their exposure to different firms within the healthcare system as well as curriculum requirements. ${ }^{8}$ Armstrong et al had demonstrated in a prospective study that the productivity of junior doctors in the emergency department was reduced in month one of the job, compared to month four. ${ }^{9}$ They also concluded that despite the level of the junior peers being similar, this impacted the productivity of their senior peers due to increased supervision particularly over the first few months into the rotation. Lack of knowledge may also coincide with productivity at the start of a job, which would also rise towards the end of four months. This could be a reason why there was no significance in results before and after education was delivered, as subsequent cohorts of doctors rotating into acute care would be unaware of the recommended practice.

For those who are discharged from accident and emergency, it is difficult for a urologist to ensure metabolic screening has been performed prior to discharge. Patients who are admitted are seen on a post-take ward round led by the most senior urologist. We found, however, that with those patients who were admitted onto wards, metabolic screening requests were not always added, or fully completed after a ward round. The responsibility should lie with the urologist to ensure that metabolic screen requests are performed, particularly in those with confirmed stones. Junior doctors on urology should also not be 
afraid to speak up when the senior urologist has made omissions inadvertently. This can be quite difficult at times; however, evidence has shown that improving non-technical ward round skills can improve task efficiency and have a positive impact on patient safety. ${ }^{10-12}$

In our study, we found that all but one stone had contained calcium oxalate. At the time of the audit, current guidelines included a bone profile and uric acid. The most recent guidelines from NICE have now changed this to serum calcium alone, as well as considering stone analysis given the high prevalence of primary hyperparathyroidism. ${ }^{6}$ This appears reasonable with our results as approximately $5 \%$ of our cohort that provided stones for analysis had no calcium in its composition at all.

There is far more computer integration with the clinical management of patients, to help reduce human errors. Many institutes around the country now incorporate electronic prescribing into clinical practice to reduce prescribing errors. ${ }^{13}$ A study by Cho et al have shown that electronic prompts for renal function prior to contrast imaging had reduced rates of contrast-induced nephropathy. ${ }^{14}$ We believe that this is a concept that can be easily incorporated into many electronic request systems, tailored towards metabolic screening prior to computed tomography imaging for renal calculi.

\section{CONCLUSION}

Improving adherence to clinical guidelines for metabolic screening has proved difficult with departmental teaching alone. Calcium at the very least should be included in screening, especially with recurrent stone formers. Computer software may be the future to improve adherence reliably through different cohorts who frequently rotate through jobs, as well as senior urologists who fail to pick up on missed requests during the post-take ward round. Nevertheless, formal faceto-face education is advocated to reinforce knowledge in junior doctors making these initial requests.

\section{REFERENCES}

1. BMJ Best Practice. Nephrolithiasis - Symptoms, Diagnosis and Treatment. 2019. Available at: https:// bestpractice.bmj.com/topics/en-us/225.
2. Balakrishnan M, Phan Y, Cobley J, Mahmalji W. Chemolysis of A Uric Acid Stone in a Horseshoe Kidney. J Endolum Endourol 2018;1(1):e37-e40.

3. Manley J, Salim F, Patterson J. Antegrade Ureteroscopy for an Obstructing Distal Ureteric Stone in a Partial Duplex System with Ectopic Distal Insertion. J Endolum Endourol 2019;2(4):e20-e22

4. Bensalah K, Tuncel A, Gupta A, Raman J, Pearle M, Lotan Y. Determinants of Quality of Life for Patients with Kidney Stones. J Urol 2008;179(6):2238-43.

5. Lotan Y. Economics and Cost of Care of Stone Disease. Adv Chron Kidney Dis 2009;16(1):5-10.

6. NICE. Recommendations: renal and ureteric stones: assessment and management: Guidance. NICE; Available at: https://www.nice.org.uk/guidance/ng118/chapter/ Recommendations\#metabolic-testing.

7. GraphPad Software. San Diego, CA. 2018. Available at: https://www.graphpad.com/.

8. National Health Service. NHS Choices. Author. Availiable at: https://foundationprogramme.nhs. uk/programmes/2-year-foundation-programme/ foundation-programme-2/

9. Armstrong P, White A, Thakore S. Senior House Officers and Foundation Year Doctors in Emergency Medicine: Do They Perform Equally? A Prospective Observational Study. Emerg Med J 2008;25(11):725-27.

10. Harvey R, Mellanby E, Dearden E, Medjoub K, Edgar S. Developing Non-Technical Ward-Round Skills. Clinical Teacher 2015;12(5):336-40.

11. Hamilton A, Kerins J, MacCrossan M, Tallentire V. Medical Students' Non-Technical Skills (Medi-StuNTS): Preliminary Work Developing A Behavioural Marker System for the Non-Technical Skills of Medical Students in Acute Care. BMJ Simulat Technol Enhanced Learn 2018;5(3):130-39.

12. Barnes T, Yu T, Webster C. Preparedness of Medical Students and Junior Doctors for Their Role as Clinical Leaders: A Systematic Review. Med Teacher 2019;1-7.

13. Bobb A, Gleason K, Husch M, Feinglass J, Yarnold P, Noskin G. The Epidemiology of Prescribing Errors. Arch Intern Med 2004;164(7):785.

14. Cho A, Lee J, Yoon J, Jang H, Huh W, Kim Y et al. Effect of an Electronic Alert on Risk of ContrastInduced Acute Kidney Injury in Hospitalized Patients Undergoing Computed Tomography. Am J Kidney Dis 2012;60(1):74-81. 